\title{
Contenido de ácido ascórbico, polifenolestotales y actividad antioxidante en capsulas de harina de cáscara del fruto de Camu camu (Myrciaria dubia hbk), bajo dos tipos de almacenamiento
}

\section{Ascorbic acid, polyphenolstotals and antioxidant activity in Camu camu (Myrciaria dubia hbk) fruit peel flour capsules, under two types of storage.}

Edgardo García Saavedra ${ }^{1}$, Caleb Leandro Laguna ${ }^{2}$, Dina Pari Quispe ${ }^{1}$, Ynés del Carmen Tavera Arévalo $^{1}$ y Manuel Mamani Flores ${ }^{1}$.

\begin{abstract}
${ }^{1}$ Universidad Nacional de Ucayali - Perú. Carretera Federico Basadre Km 5.5, Callería. Ucayali, Perú. Email: garcia_se73@hotmail.com. ORCID: https://orcid.org/0000-0001-6299-7692

${ }^{1}$ Universidad Nacional de Ucayali - Perú. Carretera Federico Basadre Km 5.5, Callería. Ucayali, Perú. Email: dina_pari@unu.edu.pe. ORCID: https://orcid.org/0000-0002-1493-79209

${ }^{1}$ Universidad Nacional de Ucayali - Perú. Carretera Federico Basadre Km 5.5, Callería. Ucayali, Perú. Email: ines_tavera@unu.edu.pe ORCID: https://orcid.org/0000-0001-7133-4030

${ }^{1}$ Universidad Nacional de Ucayali - Perú. Carretera Federico Basadre Km 5.5, Callería. Ucayali, Perú. Email: katmt23@gmail.com. ORCID: https://orcid.org/0000-0002-0431-1813

${ }^{2}$ Universidad Nacional Intercultural de la Amazonía - Perú. Carretera a San José km. 0.63, Pucallpa, Perú. Email: caleb_leandro@unia.edu.pe, Ucayali, Perú. ORCID: https://orcid.org/0000-0002-6168-1079
\end{abstract}

\section{Resumen}

Se evaluó la estabilidad del contenido de ácido ascórbico, polifenoles totales y actividad antioxidante en capsulas de harina de cáscara del fruto de Camu camu, bajo dos tipos de almacenamiento: refrigeración y medio ambiente. Las determinaciones analíticas se realizaron por métodos instrumentales, mediante métodos espectrofotométricos. Los resultados mostraron que la capsula de harina de cáscara de camu camu al inicio de la investigación contenía: Ácido ascórbico 5.128 $\pm 0,051$ $\mathrm{g} / 100 \mathrm{~g}$; polifenoles totales: $17.040 \pm 0,071 \mathrm{gEAG} / 100 \mathrm{~g}$ y una actividad antioxidante $\left(\mathrm{IC}_{50}\right.$ frente al radical DPPH): $0.550 \pm 0.013 \mathrm{mM}$ TEAC/100g. La estabilidad almacenada a temperatura de refrigeración para el ácido ascórbico fue: $\mathrm{k}=0.0054$ 1/días; $\mathrm{D}=426.40$ días; $\mathrm{t}_{1 / 2}=128.36$ días; para los polifenoles totales: $\mathrm{k}=0.0035$ 1/días; $\mathrm{D}=657.88$ días; $\mathrm{t}_{1 / 2}=198.04$ días; para la actividad antioxidante: $\mathrm{k}=0.0058$ 1/días; $\mathrm{D}=397.00$ días; $\mathrm{t}_{1 / 2}=119.51$ días. Mientras que la almacenada a temperatura ambiente, para el ácido ascórbico fue: $\mathrm{k}=0.0087$ 1/días; $\mathrm{D}=264.66$ días; $\mathrm{t}_{1 / 2}=79.67$ días; para los polifenoles totales: $\mathrm{k}=0.0046$ 1/días; $\mathrm{D}=500.56$ días; $\mathrm{t}_{1 / 2}=150.68$ días. Para la actividad antioxidante: $\mathrm{k}=0.0099$ 1/días; $\mathrm{D}=232.58$ días; $\mathrm{t}_{1 / 2}=70.01$ días. Siendo el mejor el que se almacenó a temperatura de refrigeración, disminuyendo la capacidad antioxidante a $0.276 \pm 0.003$ mM TEAC/100 g de muestra a los 119.51 días de almacenamiento.

Palabras Clave: Camu camu, cáscara, harina, cápsulas, componente bioactivo, estabilidad.

\begin{abstract}
The stability of ascorbic acid content, total polyphenols and antioxidant activity in Camu camu fruit peel flour capsules was evaluated under two types of storage: refrigerated and ambient. The analytical determinations were carried out by instrumental methods, using spectrophotometric methods. The
\end{abstract}

Downloadable from: http://revistas.unu.edu.pe

Carretera Federico Basadre $\mathrm{Km}$ 6, Dirección de Producción Intelectual

Revista de Investigación Universitaria por Universidad Nacional de Ucayali se distribuye bajo una Licencia Creative Commons Atribución-NoComercial 4.0 Internacional. 
results showed that the camu camu peel flour capsule at the beginning of the research contained: Ascorbic acid $5.128 \pm 0.051 \mathrm{~g} / 100 \mathrm{~g}$; total polyphenols: $17.040 \pm 0.071 \mathrm{gEAG} / 100 \mathrm{~g}$ and an antioxidant activity (IC50 against DPPH radical): $0.550 \pm 0.013 \mathrm{mM}$ TEAC/100g. The stability stored at refrigeration temperature for ascorbic acid was: $\mathrm{k}=0.00541$ /day; $\mathrm{D}=426.40$ days; $\mathrm{t} 1 / 2=128.36$ days; for total polyphenols: $\mathrm{k}=0.00351 /$ day; $\mathrm{D}=657.88$ days; $\mathrm{t} 1 / 2=198.04$ days; for antioxidant activity: $\mathrm{k}=0.00581 /$ day; $\mathrm{D}=397.00$ days; $\mathrm{t} 1 / 2=119.51$ days. While that stored at room temperature, for ascorbic acid was: $\mathrm{k}=0.0087$ 1/day; $\mathrm{D}=264.66$ days; $\mathrm{t} 1 / 2=79.67$ days; for total polyphenols: $\mathrm{k}=0.00461 /$ day; $\mathrm{D}=500.56$ days; $\mathrm{t} 1 / 2=150.68$ days. For antioxidant activity: $\mathrm{k}=$ $0.00991 /$ day; $\mathrm{D}=232.58$ days; $\mathrm{t} 1 / 2=70.01$ days. Being the best the one stored at refrigeration temperature, decreasing the antioxidant capacity to $0.276 \pm 0.003 \mathrm{mM} \mathrm{TEAC} / 100 \mathrm{~g}$ of sample at 119.51 days of storage.

Keywords: Camu camu, shell, flour, capsules, bioactive component, stability.

\section{Introducción}

El camu-camu (Myrciaria dubia) es un fruto de la región amazónica, natural de las márgenes inundadas(Souza et al., 2013), tiene forma globosa, superficie lisa y brillante, color rojo oscuro a negro púrpura durante la etapa de maduración y tiene un contenido de ácido ascórbico que oscila entre 800 y 6.100 mg/100 g en la pulpa fresca (Conceição et al., 2019), siendo para Brasil la fruta con el mayor contenido de vitamina $\mathrm{C}$, que tiene de 2,4 a 3,0 $\mathrm{g} / 100 \mathrm{~g}$ de pulpa, siendo un tesoro, con alto potencial socioeconómico y nutricional para la región, pero poco explorado (Pimentel et al., 2019), siendo promisorio por su principal potencial funcional (Fujita et al., 2013).

En el Perú la pulpa de camu camu procedente de los cultivos de Yarinacocha muestra alta concentración de ácido ascórbico: 1921,3 $\mathrm{mg} / 100 \mathrm{~g}$ de pulpa (García-Saavedra, Leandro-
Laguna, Vicente-Santa Cruz, Hilario, Román, \& Ruiz-Aguilar, 2012), donde la vitamina c es un micronutriente básico en la alimentación humana al estar asociada a la síntesis de diferentes moléculas de importancia en la salud humana, y su efecto antioxidante relacionado con la reducción del riesgo de contraer diferentes tipos de cáncer (Terry et al., 2019).

El camu camu además de ser fuente potencial de vitamina C (Imán et al., 2011), y de antioxidantes (Fidelis, de Oliveira, et al., 2020) nutricionales ( $\beta$-caroteno) (Chirinos et al., 2010). También posee propiedades antimicrobianas (Ruiz-barrueto et al., 2021) de protección y de regeneración celular, se han identificado compuestos fenólicos como: elagitaninos, ácido elágico, quercetina glucósidos, ácido siríngico y miricetina (Fujita et al., 2015; Schmidt et al., 2010). El alto 
contenido de vitamina $\mathrm{C}$, favorece la formación del colágeno, responsable de la formación y fortalecimiento de los huesos, músculos, tendones, ligamentos, dientes, encías, tejidos conjuntivos y vasos sanguíneos (Akter et al., 2011).

El mayor contenido de ácido ascórbico en estado fresco se presenta en la cáscara de Camu-camu madura con $21,95 \pm 1,18 \mathrm{mg} \cdot \mathrm{g}^{-1}$ de Ac. Ascórbico, seguida por el pintón 20,50 $\pm 0,39 \mathrm{mg} \cdot \mathrm{g}^{-1}$ de Ac. Ascórbico y luego la verde $13,78 \pm 1,24 \mathrm{mg} \cdot \mathrm{g}^{-1}$ de Ac. Ascórbico (Villanueva-Tiburcio, Condezo-Hoyos, \& Asquieri, 2010), siendo. Luego del secado, el mayor contenido de ácido ascórbico se presenta en la cáscara del fruto pintón con 53,49 \pm 9,40 mg. $\mathrm{g}^{-1}$ de Ac. Ascórbico, seguido por la cáscara del fruto maduro $16,41 \pm 3,64$ mg.g $\mathrm{g}^{-1}$ de Ac. Ascórbico y luego la cáscara del fruto verde $15,38 \pm 5,81 \mathrm{mg} \cdot \mathrm{g}^{-1}$ de Ac. Ascórbico, esta variación de concentración de ácido ascórbico, presenta relación con el contenido de acidez, debido a que los ácidos presentes en la fruta, facilitan la degradación del ácido ascórbico, así como la presencia de enzimas, antocianinas, paralelamente el ácido ascórbico es deteriorado por la presencia de agua, que es proporcionado por la fruta (Lee y Chen, 1998).
Sin embargo la cáscara seca del fruto maduro de camu camu, presenta una concentración de polifenoles totales con $6,02 \pm 0,48 \mathrm{mg} \cdot \mathrm{g}^{-1}$, fruto pintón 7,70 \pm 0,26 mg.. ${ }^{-1}$, fruto verde mostró la menor concentración de polifenoles totales con 5,95 $\pm 0,13 \mathrm{mg} . \mathrm{L}^{-1}$ expresado todos como ácido gálico (Villanueva-Tiburcio, Condezo-Hoyos, \& Asquieri, 2010). Otros estudios demostraron que la cáscara del Camu camu maduro contiene polifenoles: 5,52 \pm 0,39 mg.g ${ }^{-1}$ expresado como ácido gálico (García-Saavedra, Leandro-Laguna, RuizAguilar, Zorrila-Tarazona, \& Sulca Tanta, 2015). Una disminución en el contenido de compuestos fenólicos totales durante el secado podría atribuirse a la unión de polifenoles con otros compuestos (proteínas) o a alteraciones en la estructura química de los polifenoles, que no pudieron extraerse o determinarse con los métodos disponibles. (Mrad et al. citado por Zhou et al. 2016). El incremento leve en el contenido de compuestos fenólicos totales en el período de secado final, puede deberse que el largo tiempo de secado y la alta temperatura destruye la estructura celular y liberar aún más compuestos fenólicos unidos (Boudhrioua et al. citado por Zhou et al. 2016).

Asimismo, la Ea para la vitamina C, polifenoles totales y capacidad antioxidante varía entre 8.8466 y $26.5149 \mathrm{~kJ} / \mathrm{mol}$, pero es 
menor para polifenoles totales y mayor para la capacidad antioxidante. La vida útil de todos los compuestos bioactivos evaluados se reduce con el incremento de la temperatura de almacenamiento (Basilio-Atencio \& PaduroContreras, 2021).

Por otro lado, las semillas de camu-camu (Myrciaria dubia) se desechan sin recuperar los compuestos bioactivos un extracto optimizado mostró una capacidad antioxidante medida por diferentes ensayos químicos y biológicos, ejerció efectos antiproliferativos y citotóxicos contra las células cancerosas A549 y HCT8, efectos antimicrobianos, protegió los eritrocitos humanos contra la hemólisis, inhibió las enzimas $\alpha$-amilasa y $\alpha$-glucosidasa y presentó un efecto antihipertensivo in vitro. Además, el extracto optimizado inhibió la oxidación inducida por el cobre de las LDL humanas in vitro y redujo la liberación de TNF- $\alpha$ y la activación de NF- $\kappa$ B en cultivos celulares de macrófagos (Fidelis, do Carmo, et al., 2020).

De esta manera las semillas de camu-camu (Myrciaria dubia) son una rica fuente de compuestos antioxidantes antimutagénicos, citotóxicos (Carmo et al., 2019), antiinflamatorios, antimicrobianos, antihipertensivos y neuroprotectores (Do
Carmo et al., 2020), razón por la cual la cubierta de la semilla de camu-camu es un residuo industrial que puede ser explotado por sus compuestos bioactivos, lo que sugiere aplicaciones prometedoras en la tecnología alimentaria (Fidelis et al., 2018)

Por las bondades que presenta la pulpa, cascara y semilla de camu-camu (Myrciaria dubia), es importante se evaluó la estabilidad del contenido de ácido ascórbico, polifenoles totales y la capacidad antioxidante en capsulas de harina cáscara del fruto de camu camu (Myrciaria dubia HBK) bajo dos condiciones de almacenamiento: refrigeración y medio ambiente.

\section{Metodología}

Se utilizaron cáscaras de frutos maduros de camu camu, los cuales fueron obtenidos de la Asociación de Productores de camu camu el Camucamal - Ucayali, en las instalaciones de la Planta Piloto del IIAP. Donde se oreó durante cuatro horas con la finalidad de eliminar el exceso de agua, seguidamente se secó la cáscara de camu camu en un secador de lecho fluidizado a la temperatura de $70^{\circ} \mathrm{C}$ y velocidad de aire $2.5 \mathrm{~m} / \mathrm{s}$. Una vez secas las cáscaras, se procedió a reducir de tamaño en un molino de martillo para obtener la harina. 
La harina obtenida se tamizó con la finalidad de uniformizar el tamaño de las partículas, con un tamiz $\mathrm{N}^{\circ} 35(0,50 \mathrm{~mm})$ de espesor.

La harina obtenida se encapsuló y se colocó en frascos de plásticos, los mismos que fueron almacenados a temperatura ambiente $(28 \pm 2$ $\left.{ }^{\circ} \mathrm{C}\right) \quad$ y refrigeración $\left(10 \quad \pm \quad 1 \quad{ }^{\circ} \mathrm{C}\right)$ respectivamente. En el análisis del compuesto bioactivo del ácido ascórbico, se realizó mediante el método espectrofotométrico, reportado por Hung y Yen (2002), el análisis de compuestos bioactivo polifenoles totales, se realizó mediante el método espectrofotométrico.

La evaluación de la actividad antioxidante en la cápsula de harina de cascara de camu camu Radical 2,2-diphenyl-1-picrilhydracyl $(\mathrm{DPPH})$, fue realizado mediante el método descrito por Brand-Williams, Cuvelier y Berset (1995). Para la curva se utilizó stock Tolox $2 \mathrm{mM}$.

Para la determinación de los parámetros cinéticos de degradación térmica en las capsulas de harina de cascara de camu se tuvo en cuenta lo siguiente:

Constante de velocidad de degradación (k), la degradación del contenido de los compuestos bioactivos por efecto de la temperatura se representó mediante la ecuación.

$$
\operatorname{Ln}(\mathrm{C})=\operatorname{Ln}\left(\mathrm{C}_{0}\right)-\mathrm{k} . \mathrm{t}
$$

El tiempo de reducción decimal D (min), es el reciproco de la pendiente de la curva de destrucción térmica o curva de resistencia térmica. Se calculó con la siguiente ecuación:

$$
\mathrm{D}=\operatorname{Ln}(10) / \mathrm{k}
$$

Tiempo de vida media $\left(\mathrm{t}_{1 / 2}\right)$, Es el tiempo requerido para reducir el $50 \%$ de la concentración original de vitamina C. Se calcula mediante la expresión:

$$
\mathrm{t}_{1 / 2}=\operatorname{Ln}(2) / \mathrm{k}
$$

Se aplicó el diseño completo al azar (DCA), con tres repeticiones. Se aplicó la prueba comparaciones múltiples de medias de Tukey para las variables fisicoquímicas y contenido de ácido elágico, para determinar las posibles diferencias significativas entre los tratamientos.

\section{Resultados y discusiones}

Contenido de componentes bioactivos en la harina de cáscara del fruto de camu camu

El contenido de ácido ascórbico $(5.128 \pm 0.051$ $\mathrm{g} / 100 \mathrm{~g})$ o $(5128 \pm 51 \mathrm{mg} / 100 \mathrm{~g})$, presente en 648

Downloadable from: http://revistas.unu.edu.pe

Carretera Federico Basadre Km 6, Dirección de Producción Intelectual

Revista de Investigación Universitaria por Universidad Nacional de Ucayali se distribuye bajo una Licencia Creative Commons Atribución-NoComercial 4.0 Internacional. 
la harina de cáscara de camu camu mostrado en la tabla 1, es mucho mayor a los valores publicados en investigaciones, en harina de cáscara de mango variedad Tommy Atkins (332.967 $\pm 28.73 \mathrm{mg} / 100 \mathrm{~g})$ ácido ascórbico,

Tabla 1

Contenido de ácido ascórbico, polifenoles totales y actividad antioxidante de la harina de camu

camu

\begin{tabular}{lrr}
\hline \multicolumn{1}{c}{ Componente } & Unidad & Cantidad $^{\mathbf{1}}$ \\
\hline Ácido ascórbico & $\mathrm{g} / 100 \mathrm{~g}$ & $5.128 \pm 0.051$ \\
Polifenoles totales & $\mathrm{g} \mathrm{EAG} / 100 \mathrm{~g}$ & $17.040 \pm 0.071$ \\
Actividad antioxidante & $\mathrm{mM} \mathrm{TEAC} / 100 \mathrm{~g}$ & $0.550 \pm 0.013$ \\
\hline${ }^{1}$ Los valores representan la media $\pm \mathrm{SD}$, para $\mathrm{n}=3$ &
\end{tabular}

El contenido de polifenoles totales $(17.040 \pm$ $0.071 \mathrm{~g}$ EAG/100g) o $(17040 \pm 71 \mathrm{mg}$ EAG/100g) presente en la harina de cáscara de camu camu mostrado en la tabla 2 , es mucho mayor a los valores encontrados en otras investigaciones, lima dulce (C. limetta): 1.25 $\pm 0.02 \mathrm{~g}$ AGE/100 g; limón rugoso $(C$. jambhiri Lushington): $1.42 \pm 0.03$ g AGE/100 g; mandarina común (C. reticulata): $3.22 \pm$ $0.05 \mathrm{~g}$ AGE/100 g; naranja Valencia ( $C$. sinensis L. sbeck): $1.40 \pm 0.04 \mathrm{~g} \mathrm{AGE} / 100 \mathrm{~g} \mathrm{y}$ toronja (C. paradisi Macf): $3.08 \pm 0.03 \mathrm{~g}$ AGE/100 g (Ordoñez-Gómez, Reátegui-Díaz y Villanueva-Tiburcio, 2018). También encontraron fenoles totales en cáscara seca de papa: 1954,38 $\pm 0,80 \mu \mathrm{g}$ EAG/g (Arenas, 2018). variedad Keitt $(338.095 \pm 21.48 \mathrm{mg} / 100 \mathrm{~g})$ ácido ascórbico, variedad Criollo (512.637 \pm $59.97 \mathrm{mg} / 100 \mathrm{~g}$ ) ácido ascórbico (Serna-Cock, Torres-León y Ayala-Aponte, 2015). 
UNIVERSIDAD NACIONAL DE UCAYALI

Revista de Investigación Universitaria

Versión electrónica 2664 - 8423

ARTICULO ORIGINAL
Vol. $11 \mathrm{~N}^{\circ}$ 2, pp. 644 - 663, julio/diciembre 2021

Recibido 29/09/2021

Aceptado 19/10/2021

Publicado 30/12/2021 camu camu es mayor a la capacidad antioxidante de la kiwicha: $1.2 \pm 0.01$ umol TE/g; quinua: $5.3 \pm 0.01$ umol TE/g y cáscara de haba: $1.2 \pm 0.01 \mathrm{umol} \mathrm{TE} / \mathrm{g}$, pero es menor a la capacidad antioxidante de la haba entera seca: $7.1 \pm 0.06$ umol TE/g (Ramos, 2011).

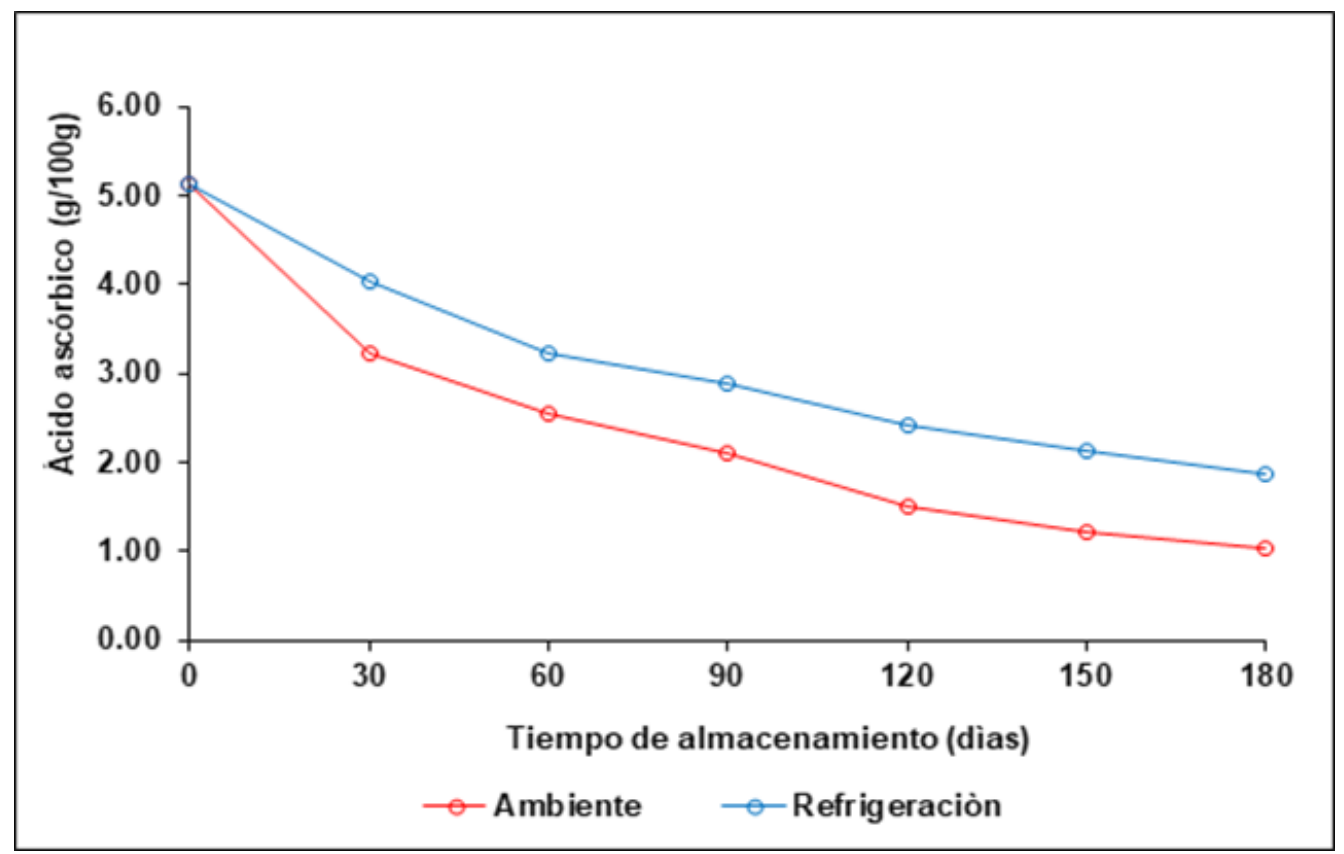

Figura 1: Variación del contenido de ácido ascórbico $(\mathrm{g} / \mathrm{l} / 00 \mathrm{~g})$ durante el almacenamiento.

La diferencia en el contenido de componentes bioactivos y en la capacidad antioxidante se explica por las condiciones agroecológicas y el manejo agronómico de los cultivos de camu camu que son factores que se integran ye interactúan en el desarrollo vegetativo y reproductivo de las plantas.

\section{Estabilidad de los componentes bioactivos durante el almacenamiento}

Para determinar la estabilidad o cinetica de degradación de los componentes bioactivos, los datos de cada componente, se sometieron a un análisis de regresión con los modelos de cinética de orden cero, de primer orden y de segundo orden.

La Figura 1 muestra el comportamiento de degradación del contenido de vitamina $\mathrm{C}$, bajo las dos condiciones de almacenamiento conde se observa que, a mayor concentración de ácido ascórbico, menor será en tiempo de conservación en ambos métodos.

Es decir que existe mayor pérdida de vitamina $\mathrm{C}$ ocurre durante los primeros 30 
días, siendo más pronunciado en el almacenamiento a temperatura ambiente $\left(28 \pm 2^{\circ} \mathrm{C}\right)$. Durante el periodo de almacenamiento (180 días) la menor pérdida de ácido ascórbico se dio en las capsulas almacenadas en refrigeración (10 $\left.\pm 1^{\circ} \mathrm{C}\right)$.

La degradación del ácido ascórbico depende de varios factores: temperatura, oxígeno, catálisis de iones metálicos, luz, humedad, etc. (Fennema, 2000; Gamboa-Santos et al. 2014). El ácido ascórbico es el principal nutriente en la cáscara de camu camu, y se degrada rápidamente. Si tenemos en cuenta la ingesta diaria de referencia (RDI) para el ácido ascórbico, $60 \mathrm{mg}$, según lo establecido por la Administración de Drogas y Alimentos de los Estados Unidos (Lee y Chen 1998), podemos afirmar que, la harina de cáscara de camu camu en cápsulas, al final del periodo de almacenamiento (180 días) contiene: $1.04 \mathrm{~g}$ de ácido ascórbico/100 g de harina o su equivalente $1041.00 \mathrm{mg}$ de vitamina $\mathrm{C} / 100 \mathrm{~g}$ de harina para las cápsulas almacenadas a temperatura ambiente, y $1.88 \mathrm{~g}$ de ácido ascórbico/100 g de harina $1878.27 \mathrm{mg}$ de vitamina $\mathrm{C} / 100 \mathrm{~g}$ de harina para las cápsulas almacenadas en refrigeración. Lo que lo hace atractivo como producto aún al someter a condiciones de almacenamiento no tan adecuado.

Existe un efecto de la temperatura de almacenamiento en la pérdida de ácido ascórbico en las capsulas de harina de cáscara de camu camu. La pérdida de ácido ascórbico es mayor cuando las cápsulas se almacenan a temperatura ambiente. Esto es confirmado por una investigación previa que un almacenamiento a menor temperatura podría disminuir la tasa de degradación de la vitamina C (Sapei y Hua 2014), y que fue corroborado en la investigación para las cápsulas almacenadas a temperatura de refrigeración.

La vitamina $\mathrm{C}$ exhibió mayor estabilidad en el producto de mango en polvo almacenado a una temperatura de $4^{\circ} \mathrm{C}$. Presentó una cinética de degradación de primer orden. Las mayores variaciones se dieron en las muestras almacenadas a $28^{\circ} \mathrm{C}$, lo cual indica la influencia de la temperatura en el cambio de los componentes del producto (MendozaCorvis et al., 2017). 


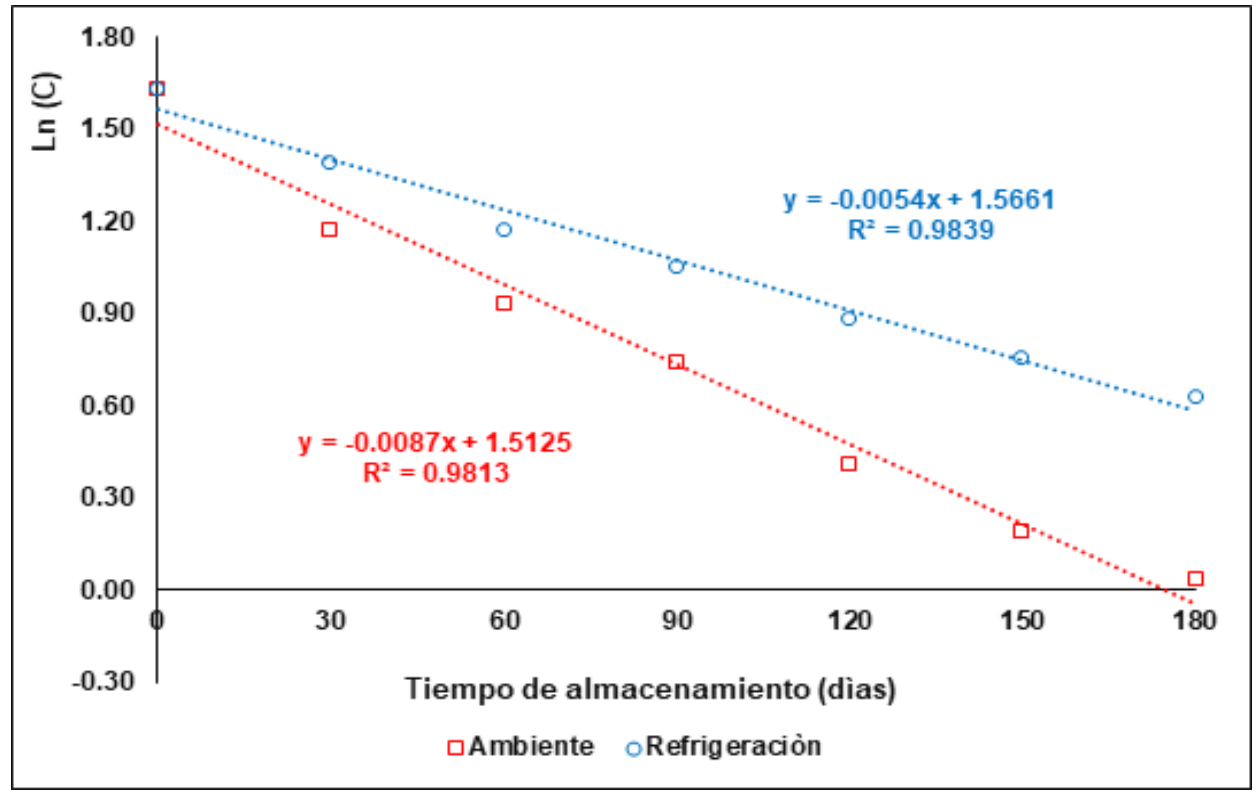

Figura 2: Cinética de degradación de primer orden para el contenido de ácido ascórbico, bajo las dos condiciones de almacenamiento.

Por otro lado, en la figura 2, se muestra la cinética de degradación de la vitamina C la misma que es descrita mediante un modelo de reacción de primer orden, ya que presentaron coeficientes de determinación $\left(\mathrm{R}^{2}\right)$ superiores a 98\%. La velocidad de degradación depende de la temperatura, porque el aumento de la temperatura de almacenamiento reportó un valor mayor en la velocidad de degradación. Lo anterior pone de manifiesto el aumento de la velocidad de degradación de la vitamina $\mathrm{C}$ con el incremento de la temperatura.

Degradación del contenido de polifenoles totales 


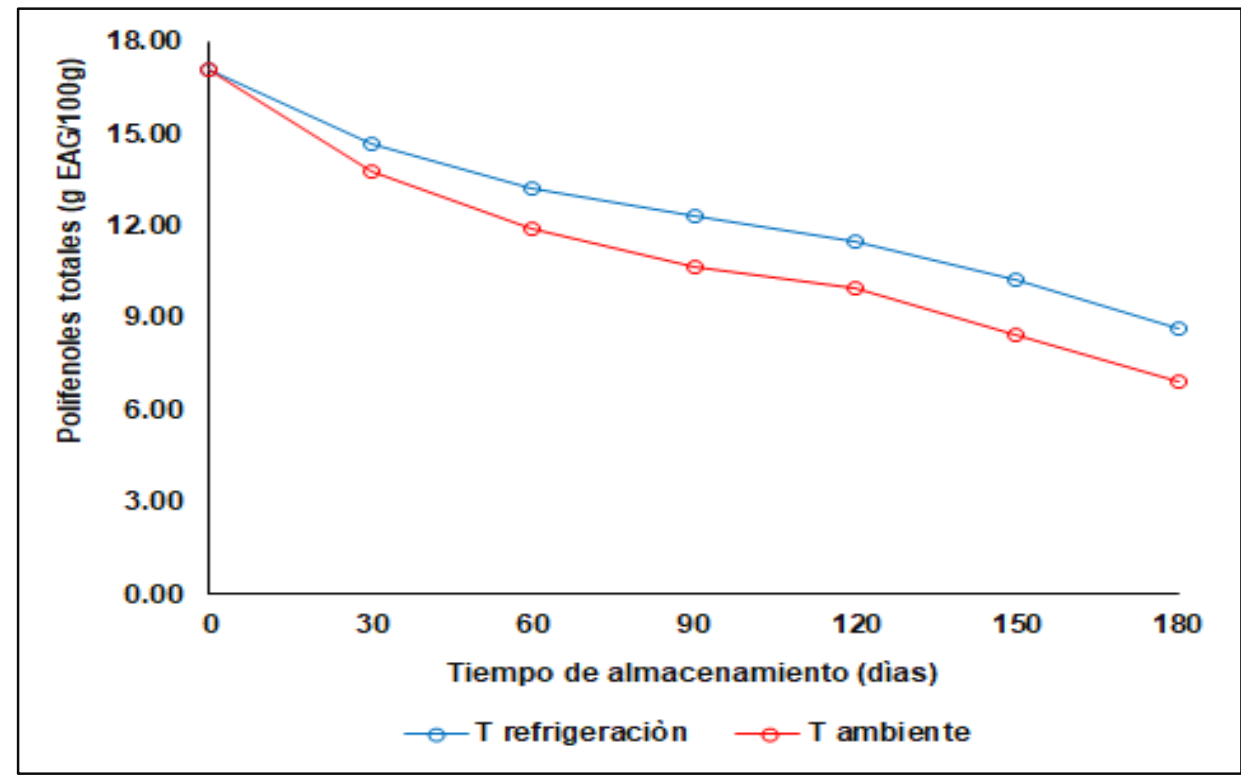

Figura 3: Variación del contenido de polifenoles totales durante el almacenamiento, expresado en $g$ EAG/100g (Equivalentes de ácido elágico). A temperatura ambiente y refrigeración.

En la Figura 4 se observa que la mayor pérdida de polifenoles totales ocurre durante los primeros 30 días (período donde el gráfico presenta mayor pendiente), es menos pronunciado cuando el almacenamiento fue a temperatura de refrigeración $\left(10 \pm 1^{\circ} \mathrm{C}\right)$. Durante el periodo de almacenamiento (180 días) se observó que el contenido de polifenoles totales disminuye conforme el tiempo de almacenamiento se incrementa, siendo mayor la pérdida de polifenoles totales de la harina de camu camu encapsulada aquellas que fueron almacenadas a temperatura ambiente $\left(28 \pm 2{ }^{\circ} \mathrm{C}\right)$.

En estudios reportados se observaron que la degradación de polifenoles totales en la bebida a base de arándano siguió una cinética de primer orden a todas las temperaturas de almacenamiento 30,40 y $50^{\circ} \mathrm{C}$ (Torres y Vidaurre, 2015). También, la variación en el contenido de polifenoles totales durante el almacenamiento de la bebida de borojó presentó una cinética de primer orden. La mayor velocidad de degradación se obtuvo para la temperatura de almacenamiento de $37^{\circ} \mathrm{C}$ (Camelo-Méndez y Sotelo, 2012). El almacenamiento de la bebida de borojó a $4^{\circ} \mathrm{C}$ permitió una mejor retención en el contenido de polifenoles (Camelo-Méndez y Sotelo, 2012). 


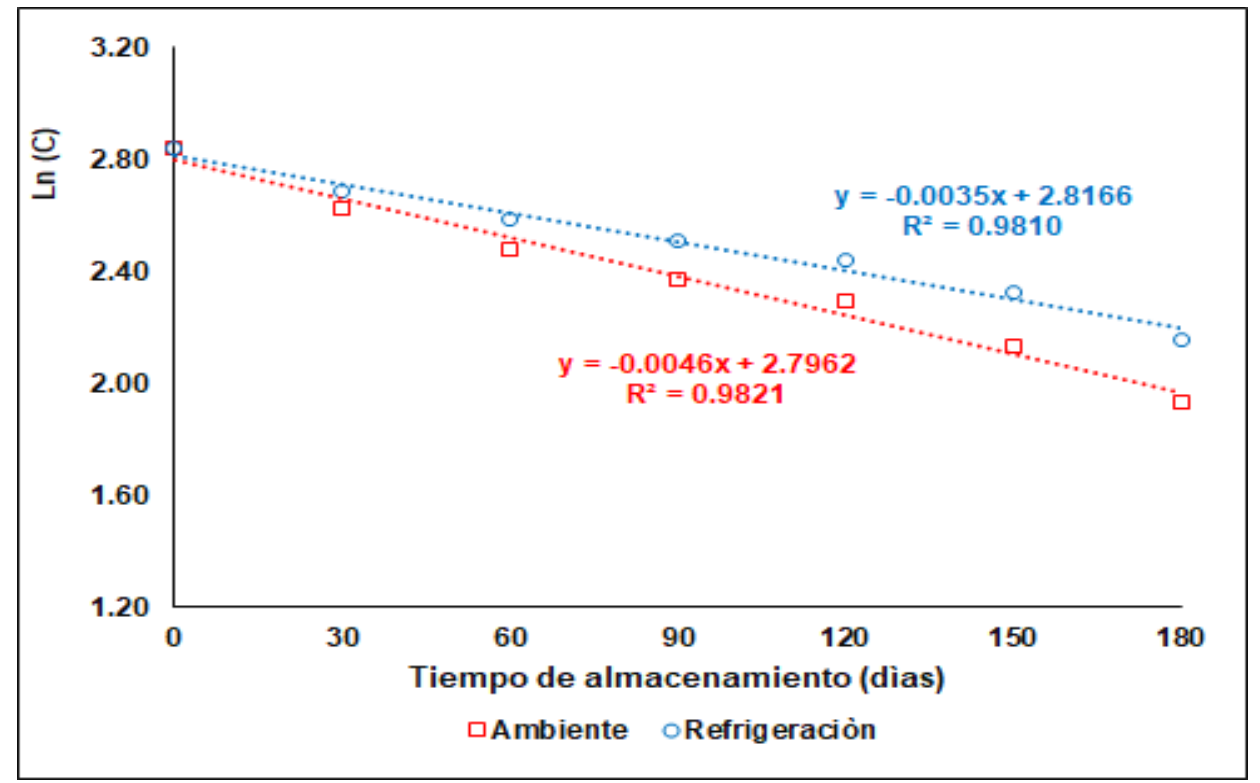

Figura 4: Cinética de degradación de primer orden para el contenido de polifenoles totales de las cápsulas de cати сати, durante el almacenamiento.

Degradación de la actividad antioxidante

El modelo de reacción de primer orden describió adecuadamente la cinética de degradación para la actividad antioxidante de la harina de cáscara de camu camu en cápsulas, ya que el coeficiente de determinación $\left(\mathrm{R}^{2}\right)$ fue superior a 98\%, para ambas condiciones de almacenamiento. Las constantes de velocidad de degradación dependen de la temperatura, porque al ser la temperatura ambiente mayor se observó un mayor valor en la velocidad de degradación. Esto corrobora el aumento de la velocidad de degradación de la capacidad antioxidante con el incremento de la temperatura de almacenamiento. 
UNIVERSIDAD NACIONAL DE UCAYALI

Revista de Investigación Universitaria

Versión electrónica 2664 - 8423

ARTICULO ORIGINAL
Vol. $11 \mathrm{~N}^{\circ} 2$, pp. $644-663$, julio/diciembre 2021

Recibido 29/09/2021

Aceptado 19/10/2021

Publicado 30/12/2021

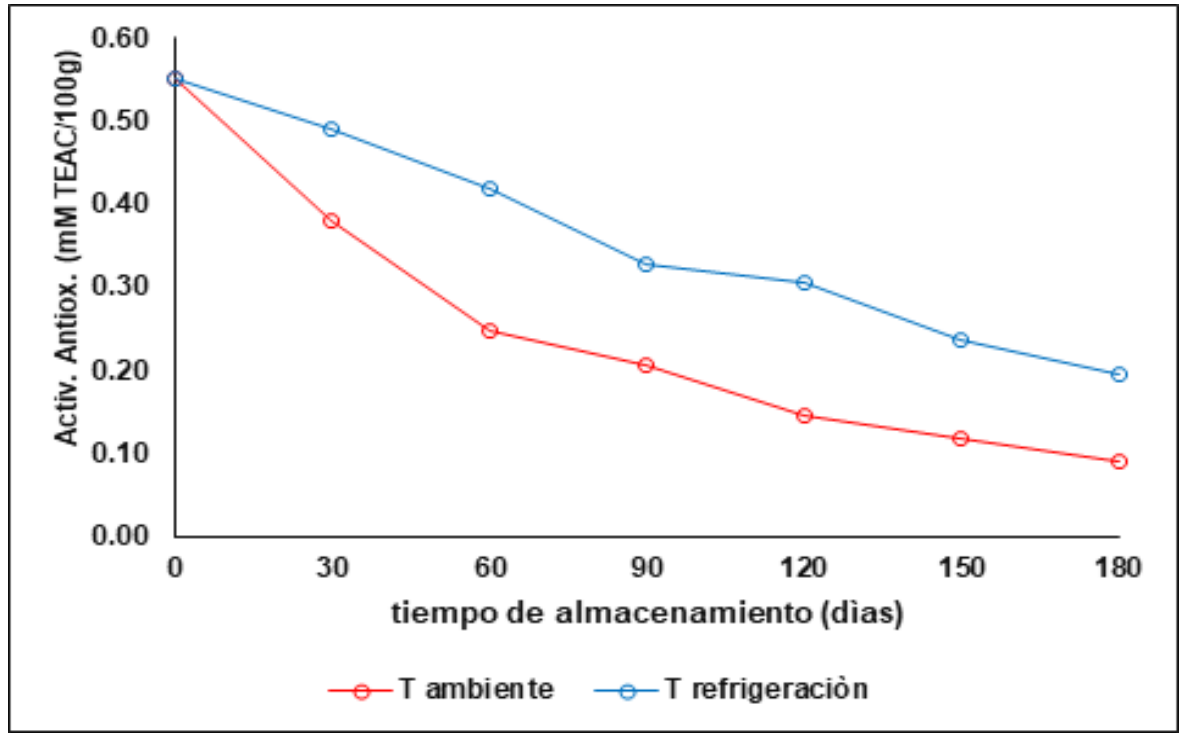

Figura 5: Variación de la actividad antioxidante durante el almacenamiento, expresados en nM

$$
\text { TEAC/100g. }
$$

En otros estudios se encontraron que después de 40 días de almacenamiento de la bebida (polvo de berenjena), se detectó una disminución de la capacidad antioxidante de la bebida (Arrazola, Herazo y Alvis, 2014).

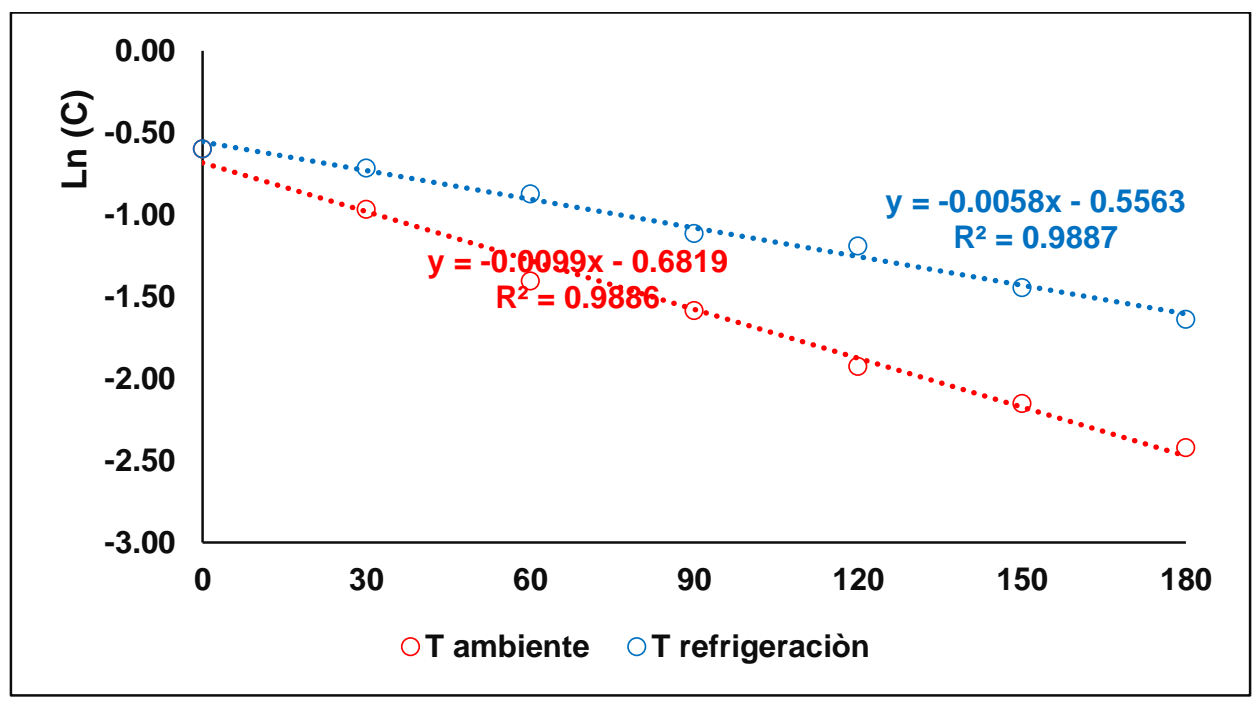

Figura 6: Cinética de degradación de primer orden para la actividad antioxidante de las cápsulas de camu camu, durante el almacenamiento

Downloadable from: http://revistas.unu.edu.pe

Carretera Federico Basadre Km 6, Dirección de Producción Intelectual

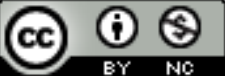

Revista de Investigación Universitaria por Universidad Nacional de Ucayali se distribuye bajo una Licencia Creative Commons Atribución-NoComercial 4.0 Internacional. 
Se encontró también que la variación en la actividad antioxidante durante el almacenamiento de la bebida de borojó presentó una cinética de primer orden; la mayor velocidad de degradación para la capacidad antioxidante fue para la temperatura de almacenamiento de $37^{\circ} \mathrm{C}$, pero el almacenamiento de la bebida de borojó almacenada a $4^{\circ} \mathrm{C}$, permitió una mejor retención de la actividad antioxidante, en comparación con las demás temperaturas de almacenamiento (Camelo-Méndez y Sotelo, 2012).

La disminución en la actividad antioxidante puede ser debidos a que compuestos inhibidores de radicales libres DPPH son susceptibles a la oxidación en condiciones de almacenamiento (Wu et al., 2010). La capacidad antioxidante dependiente del contenido de fenoles totales, en donde los productos con alto contenido de polifenoles poseen una alta capacidad antioxidante, asimismo, las temperaturas elevadas de almacenamiento podrían acelerar la degradación oxidativa de los polifenoles y de esta forma la actividad (Camelo-Méndez y Sotelo, 2012).

Parámetros cinéticos de la degradación de compuestos bioactivos

\section{Tabla 2}

Velocidad de degradación ( $k$ ) y coeficiente de determinación $\left(R^{2}\right)$ para los compuestos bioactivos de la harina de cáscara de camu camu.

\begin{tabular}{|c|c|c|c|c|c|c|}
\hline \multicolumn{7}{|c|}{ Para el contenido de ácido ascórbico } \\
\hline \multirow{2}{*}{$\begin{array}{l}\text { Condiciones de } \\
\text { almacenamiento }\end{array}$} & \multicolumn{2}{|c|}{ Reacción de orden cero } & \multicolumn{2}{|c|}{$\begin{array}{c}\text { Reacción de primer } \\
\text { orden }\end{array}$} & \multicolumn{2}{|c|}{$\begin{array}{c}\text { Reacción de segundo } \\
\text { orden }\end{array}$} \\
\hline & $\mathbf{k}_{\mathbf{0}}$ & $\mathbf{R}^{2}$ & $\mathbf{k}_{1}$ & $\mathbf{R}^{2}$ & $\mathbf{k}_{2}$ & $\mathbf{R}^{2}$ \\
\hline $\mathrm{T}_{\text {ambiente }}$ & 0.0207 & 0.8738 & 0.0087 & 0.9813 & 0.0043 & 0.9812 \\
\hline $\mathrm{T}_{\text {refrigeración }}$ & 0.0171 & 0.9279 & 0.0054 & 0.9839 & 0.0019 & 0.9975 \\
\hline \multicolumn{7}{|c|}{ Para el contenido de polifenoles totales } \\
\hline \multirow{2}{*}{$\begin{array}{l}\text { Condiciones de } \\
\text { almacenamiento }\end{array}$} & \multicolumn{2}{|c|}{ Reacción de orden cero } & \multicolumn{2}{|c|}{$\begin{array}{c}\text { Reacción de primer } \\
\text { orden }\end{array}$} & \multicolumn{2}{|c|}{$\begin{array}{c}\text { Reacción de segundo } \\
\text { orden }\end{array}$} \\
\hline & $\mathbf{k}_{\mathbf{0}}$ & $\mathbf{R}^{2}$ & $\mathbf{k}_{1}$ & $\mathbf{R}^{2}$ & $\mathbf{k}_{2}$ & $\mathbf{R}^{2}$ \\
\hline $\mathrm{T}$ ambiente & 0.0513 & 0.9546 & 0.0046 & 0.9821 & 0.0004 & 0.9626 \\
\hline $\mathrm{T}_{\text {refrige }}$ & 0.0427 & 0.9736 & 0.0035 & 0.9810 & 0.0003 & 0.9606 \\
\hline \multirow{3}{*}{$\begin{array}{l}\text { Condiciones de } \\
\text { almacenamiento }\end{array}$} & \multicolumn{4}{|c|}{ Para la actividad antioxidante } & \multirow{2}{*}{\multicolumn{2}{|c|}{$\begin{array}{c}\text { Reacción de segund } \\
\text { orden }\end{array}$}} \\
\hline & \multicolumn{2}{|c|}{ Reacción de orden cero } & \multicolumn{2}{|c|}{$\begin{array}{c}\text { Reacción de primer } \\
\text { orden }\end{array}$} & & \\
\hline & $\mathbf{k}_{\mathbf{0}}$ & $\mathbf{R}^{2}$ & $\mathbf{k}_{1}$ & $\mathbf{R}^{2}$ & $\mathbf{k}_{2}$ & $\mathbf{R}^{2}$ \\
\hline $\mathrm{T}$ ambiente & 0.0024 & 0.8826 & 0.0099 & 0.9886 & 0.0511 & 0.9669 \\
\hline
\end{tabular}




\begin{tabular}{lllllll}
\hline $\mathrm{T}_{\text {refrigeración }}$ & 0.0020 & 0.9859 & 0.0058 & 0.9887 & 0.0182 & 0.9497 \\
\hline
\end{tabular}

Para determinar el orden de reacción y las constantes de velocidad de los compuestos estudiados, se realizó un análisis de regresión lineal simple. Para los modelos cinéticos indicados en la metodología. Luego de aplicar la ecuación 1 de reacción de primer orden para tratar de conocer la cinética de degradación de la vitamina $\mathrm{C}$, se obtuvieron los parámetros cinéticos, velocidad degradación (k), tiempo de reducción decimal (D) mediante la ecuación 2 , tiempo de vida media $\left(\mathbf{t}_{1 / 2}\right)$ mediante la ecuación 3, para la harina de cáscara de camu camu en cápsulas (tabla 3).

Tabla 3

Parámetros cinéticos obtenidos para la reacción de primer orden

\begin{tabular}{lcccc}
\hline Compuesto bioactivo & $\begin{array}{c}\text { Condicione de } \\
\text { almacenamiento }\end{array}$ & $\begin{array}{c}\mathbf{k} \\
\text { (1/días) }\end{array}$ & $\begin{array}{c}\mathbf{D} \\
\text { (días) }\end{array}$ & $\begin{array}{c}\mathbf{t}_{\mathbf{1} 2 \mathbf{2}} \\
\text { (días) }\end{array}$ \\
\hline \multirow{2}{*}{ Ácido ascórbico } & Ambiente & 0.0087 & 264.66 & 79.67 \\
& Refrigeración & 0.0054 & 426.40 & 128.36 \\
Polifenoles totales & Ambiente & 0.0046 & 500.56 & 150.68 \\
\multirow{2}{*}{ Actividad antioxidante } & Refrigeración & 0.0035 & 657.88 & 198.04 \\
& $\begin{array}{c}\text { Ambiente } \\
\text { Refrigeración }\end{array}$ & 0.0099 & 232.58 & 70.01 \\
& & 0.0058 & 397.00 & 119.51 \\
\hline
\end{tabular}

Los parámetros cinéticos de degradación (Tabla 3) fueron calculados a partir de las reacciones de primer orden para los componentes bioactivos presentes en la capsula de harina de cáscara de camu camu.

La velocidad de degradación de los componentes bioactivos presentes en las capsulas de harina de cascara de camu camu fue mayor en las almacenadas a temperatura ambiente. Las constantes de velocidad cinética del mango en polvo almacenado a diferentes temperaturas, dependen estrechamente de ésta, el aumento en la temperatura de almacenamiento implicó un valor mayor en la constante de velocidad de degradación (Mendoza-Corvis et al., 2017).

La temperatura de almacenamiento en bebidas ejerce una influencia significativa en la degradación de las antocianinas, siendo la temperatura ambiente de $25^{\circ} \mathrm{C}$ la que presentó los menores valores de vida media y porcentajes de retención (Arrazola, Herazo y Alvis, 2014). Los valores de las constantes cinéticas (k) para el contenido de polifenoles 
totales y capacidad antioxidante varían con el incremento de la temperatura y tiempo de almacenamiento de la bebida de borojó (Camelo-Méndez y Sotelo, 2012).

Similar comportamiento se obtuvo para las cápsulas de harina de cáscara de camu camu, se observó que a menor temperatura (refrigeración: $9 \pm 1{ }^{\circ} \mathrm{C}$ ) el tiempo de vida media es mayor, para todos los componentes bioactivos. En la bebida de arándano los compuestos fenólicos se degradaron a una constante de velocidad de 0,0004; 0,0009 y 0,0017 , a las temperaturas de 50,40 y $30^{\circ} \mathrm{C}$ respectivamente (Torres y Vidaurre, 2015).

Las constantes de velocidad varían de acuerdo a la temperatura, a mayor temperatura mayor será su constante de velocidad. (Garzón, 2008).

\section{Conclusiones}

Las capsulas de harina de cáscara de camu camu, presentan un contenido de ácido ascórbico: $5.128 \pm 0.051 \mathrm{~g}$ AA/100 g, polifenoles totales: $17.040 \pm 0.071 \mathrm{~g}$ de EAG/100 g, capacidad antioxidante: $0.550 \pm$ $0.013 \mathrm{mM}$ TEAC/100 $\mathrm{g}$ de muestra. Los parámetros cinéticos de degradación de primer orden nos muestran la estabilidad de los componentes bioactivos en las capsulas de harina de cáscara de camu camu, almacenadas a temperatura de refrigeración y a temperatura ambiente respectivamente: ácido ascórbico: $\mathbf{k}$ $=0.0054$ 1/días; $\mathbf{k}=0.0087$ 1/días $\mathbf{D}=426.40$ días; $\mathbf{D}=264.66$ días $\mathbf{t}_{1 / 2}=128.36$ días, $\mathbf{t}_{1 / 2}=$ 79.67 días. Para los polifenoles totales: $\mathbf{k}=$ 0.0035 1/días; $\mathbf{k}=0.0046$ 1/días $\mathbf{D}=657.88$ días; $\mathbf{D}=500.56$ días $\mathbf{t}_{1 / 2}=198.04$ días, $\mathbf{t}_{1 / 2}=$ 150.68 días. Para la actividad antioxidante: $\mathbf{k}$ $=0.0058$ 1/días; $\mathbf{k}=0.0099$ 1/días $\mathbf{D}=397.00$ días; $\mathbf{D}=232.58$ días $\mathbf{t}_{\mathbf{1} / 2}=119.51$ días, $\mathbf{t}_{\mathbf{1} / \mathbf{2}}=$ 70.01 días. Un adecuado almacenamiento de las cápsulas de cáscara de harina de camu camu es a refrigeración, porque tiene una baja constante de velocidad de degradación $(\mathbf{k}=$ 0.0099 1/días); además, el tiempo de vida media $\left(\mathbf{t}_{1 / 2}=119.51\right.$ días $)$ fue mayor y su capacidad antioxidante a $0.276 \pm 0.003 \mathrm{mM}$ TEAC/100 g de muestra ha disminuido a los 119.51 días de almacenamiento.

\section{Referencias bibliográficas}

Akter, S.; Oh, S.; Bang, J.; Ahmed, M. 2011. Nutritional compositions and health promoting phytochemicals of camucamu (Myrciaria dubia) fruit: A review. Food Research International 44(7): $1728-1732$.

Arenas, ADA. 2018. Efecto de dos tipos de secado en los compuestos antioxidantes 
de cáscara de papa (Solanum

tuberosum) variedad Diacol capiro para

la fabricación de una infusión. Tesis presentada para optar el título de ingeniero de Alimentos. Facultad de Ingeniería, Universidad de La Salle. Consultado ene. 20 de 2020. https://ciencia.lasalle.edu.co/cgi/viewc ontent.cgi?article $=1166 \&$ context $=$ ing alimentos

Arrazola, G; Herazo, I; Alvis, A. 2014. Obtención y evaluación de la estabilidad de antocianinas de berenjena (Solanum melongena L.) en bebidas. Información Tecnológica, 25(3): 43 52.

Basilio-Atencio, J. E., \& Paduro-Contreras, A. (2021). Stability evaluation by accelerated tests, of the camu camu husk ( Myrciaria dubia Mc Vaugh ) dried by hot air. Agropecuaria Science and Biotecnology, 01(02), 22-41. https://doi.org/dx.doi.org/10.25127/riagr op. 20212.676

Brand-Williams W, Cuvelier ME, Berset C. (1995). Use of a free radical method to evaluate antioxidant activity. Food Science and Technology, 28(1): 25-30.

Carmo, M. A. V. Do, Fidelis, M., Pressete, C. G., Marques, M. J., Castro-Gamero, A.
M., Myoda, T., Granato, D., \& Azevedo, L. (2019). Hydroalcoholic Myrciaria dubia (camu-camu) seed extracts prevent chromosome damage and act as antioxidant and cytotoxic agents. Food Research International, 125(July), 108551.

https://doi.org/10.1016/j.foodres.2019.1

08551

Conceição Sarmento, R., Machado Pinto, M. R., Silva Craveiro, R., Brasil De Freitas, E. P., Modesto Junior, E. N., \& Vitti Mota, R. (2019). Estabilidade do ácido ascórbico em iogurte de leite de búfala adicionado de diferentes concentrações de polpa de camu-camu (Myrciaria dubia). Revista Do Instituto de Laticínios Cândido Tostes, 74(3), 149-158. https://doi.org/10.14295/2238-

6416.v74i3.731

Camelo-Méndez, GA; Sotelo, DLI. 2012. Efecto de las condiciones de almacenamiento sobre el color, contenido de polifenoles y capacidad antioxidante de una bebida de Borojoa patinoi Cuatrecasas. Boletín Latinoamericano y del Caribe de Plantas Medicinales y Aromáticas, 11(2): 196 - 205. 
Chirinos, R.; Galarza, J.; Betalleluz-Pallardel, I.; Pedreschi, R.; Campos, D. 2010. Antioxidant compounds and antioxidant capacity of Peruvian camu-camu (Myrciaria dubia H.B.K. McVaugh) fruit at different maturity stages. Food chemistry 120(4): 1019-1024

do Carmo, M. A. V., Fidelis, M., Sanchez, C. A., Castro, A. P., Camps, I., Colombo, F. A., Marques, M. J., Myoda, T., Granato, D., \& Azevedo, L. (2020). Camu-camu (Myrciaria dubia) seeds as a novel source of bioactive compounds with promising antimalarial and antischistosomicidal properties. Food Research International, 136(February), 109334. https://doi.org/10.1016/j.foodres.2020.1 09334

Fennema O. Química de alimentos. 2nd ed. Zaragoza: Acribia. 2000.

Fidelis, M., de Oliveira, S. M., Sousa Santos, J., Bragueto Escher, G., Silva Rocha, R., Gomes Cruz, A., Araújo Vieira do Carmo, M., Azevedo, L., Kaneshima, T., Young Oh, W., Shahidi, F., \& Granato, D. (2020). From byproduct to a functional ingredient: Camu-camu (Myrciaria dubia) seed extract as an antioxidant agent in a yogurt model. Journal of Dairy Science, 103(2), 1131-
1140. https://doi.org/10.3168/jds.201917173

Fidelis, M., do Carmo, M. A. V., da Cruz, T. M., Azevedo, L., Myoda, T., Miranda Furtado, M., Boscacci Marques, M., Sant'Ana, A. S., Inês Genovese, M., Young Oh, W., Wen, M., Shahidi, F., Zhang, L., Franchin, M., de Alencar, S. M., Luiz Rosalen, P., \& Granato, D. (2020). Camu-camu seed (Myrciaria dubia) - From side stream to an antioxidant, antihyperglycemic, antiproliferative, antimicrobial, antihemolytic, anti-inflammatory, and antihypertensive ingredient. Food Chemistry, 310(May 2019), 125909. https://doi.org/10.1016/j.foodchem.2019 .125909

Fidelis, M., Santos, J. S., Escher, G. B., Vieira do Carmo, M., Azevedo, L., Cristina da Silva, M., Putnik, P., \& Granato, D. (2018). In vitro antioxidant and antihypertensive compounds from camucamu (Myrciaria dubia McVaugh, Myrtaceae) seed coat: A multivariate structure-activity study. Food and Chemical Toxicology, 120(July), 479490.

https://doi.org/10.1016/j.fct.2018.07.043 Fujita, A., Borges, K., Correia, R., Franco, B. 
D. G. de M., \& Genovese, M. I. (2013).

Impact of spouted bed drying on bioactive compounds, antimicrobial and antioxidant activities of commercial frozen pulp of camu-camu (Myrciaria dubia Mc. Vaugh). Food Research International, 54(1), 495-500. https://doi.org/10.1016/j.foodres.2013.0 7.025

Fujita, A., Sarkar, D., Wu, S., Kennelly, E., Shetty, K., \& Inés, M. (2015). Evaluation of phenolic-linked bioactives of camucamu ( Myrciaria dubia Mc . Vaugh) for antihyperglycemia , antihypertension , antimicrobial properties and cellular rejuvenation. Food Research International, $\quad 77, \quad$ 194-203. https://doi.org/10.1016/j.foodres.2015.0 7.009

Gamboa-Santos, J; Megías-Pérez, R; Soria, AC; Olano, A; Montilla, A; Villamiel, M. 2014. Impact of processing conditions on the kinetic of vitamin $\mathrm{C}$ degradation and 2-furoylmethyl amino acid formation in dried strawberries. Food Chemistry, 153(2014): 164-170.

García-Saavedra, E, Leandro-Laguna, C., Vicente E, Hilario J, Román W, Ruiz F. Variación de las características biométricas, físicas y químicas de la fruta de camu camu (Myrciaria dubia Mc. Vaugh), en tres estados de madurez: verde, pintón y maduro, procedente de los cultivos de Yarinacocha y Pucalpillo. Universidad Nacional de Ucayali. Ucayali, Perú; 2012.

García-Saavedra, S.E., Leandro-Laguna, C., Sulca-Tanta, O.H., Elaboración de una bebida isotónica a partir del extracto del desecho agroindustrial (cáscara) de camu camu (Myrciaria dubia Mc. Vaugh). Universidad Nacional de Ucayali. Ucayali, Perú; 2015.

Garzón, GA. 2008. Las antocianinas como colorantes naturales y compuestos bioactivos: Revisión. Acta biol. Colomb., 13(39: 27 - 36.

Hung C, Yen GC. Antioxidant Activity of Phenolic Compounds Isolated from Mesona Procumbens Hemsl. Journal of Agricultural and Food Chemistry. 2002; 50(10): 2993 -2997.

Imán, S.; Bravo, L.; Sotero, V.; Oliva, C. 2011. Contenido de vitamina $\mathrm{C}$ en frutos de camu-camu Myrciaria dubia (H.B.K) Mc Vaugh, en cuatro estados de maduración, procedentes de la Colección de Germoplasma del INIA Loreto, Perú. Scientia Agropecuaria 
2(3): 123-130.

Lee, HS; Chen, CS. 1998. Rates of vitamin C loss and discoloration in clear orange juice concentrate during storage at temperature of $4-24^{\circ} \mathrm{C}$. Journal of Agricultural and Food Chemistry, 46(11): 4723 - 4727.

Mendoza-Corvis, F.A., Arteaga-Márquez, M.R., Pérez-Sierra, O.A. 2017. Degradación de la vitamina $\mathrm{C}$ en un producto de mango (Mangifera indica L.) y lactosuero. Corpoica Cienc Tecnol Agropecuaria, Mosquera (Colombia), 18(1):125-137.

Pimentel, C. É. M., Santiago, I. L., Oliveira, S. K. M. S., \& Serudo, R. L. (2019). Produção de cerveja artesanal com adição de ácido ascórbico a partir de fruto amazônico. Brazilian Journal of Development, 5(10), 18553-18560. https://doi.org/10.34117/bjdv5n10-107

Ramos, C.R.A. 2011. Evaluación de la capacidad antioxidante de productos tradicionales de la región Junín "granadilla, guinda, habas, quiwicha, oca, quinua, tuna, tumbo y yacón. Consultado junio 10 de 2020. Disponible en http://repositorio.uncp.edu.pe/bitstream /handle/UNCP/1219/TESIS\%20RICA
RDO\%20A.\%20RAMOS\%20CRISPI

N.pdf?sequence $=4 \&$ isAllowed $=y$

Ruiz-barrueto, M. A., Gustavo, C., Pérez, P., \& Beatriz, P. (2021). Actividad antibacteriana in vitro del extracto hidroetanólico de Myrciaria dubia ( Kunth) McVaugh ( camu camu) sobre Streptococcus mutans In vitro antibacterial activity of hydroethanolic extract from Myrciaria dubia ( Kunth) McVaugh ( сати сати ) agai. 73(2), 111.

Sapei, L; Hwa, L. 2014. Study on the Kinetics of Vitamin C Degradation in Fresh Strawberry Juices. Procedia Chemistry, 9 (2014): $62-68$.

Serna-Cock, L; Torres-León, C; AyalaAponte, A. 2015. Evaluación de polvos alimentarios obtenidos de cáscaras de mango (Mangifera indica) como fuente de ingredientes funcionales. Información Tecnológica, 26(2): 41 50.

Schmidt, A.; Lajolo, F.; Genovese, M. 2010. Chemical composition and antioxidant/antidiabetic potential of Brazilian native fruits and commercial frozen pulps. Journal of Agricultural and Food Chemistry 58(8): 4666-4674.

Souza, A. L. R., Pagani, M. M., Dornier, M., 
Gomes, F. S., Tonon, R. V., \& Cabral, L.

M. C. (2013). Concentration of camucamu juice by the coupling of reverse osmosis and osmotic evaporation processes. Journal of Food Engineering, 119(1),

https://doi.org/10.1016/j.jfoodeng.2013.

05.004

Terry Calderón, V. M., Taramona Ruiz, L., Candela Diaz, J., \& Gonzales Requejo, J. (2019). Tiempo de reducción decimal para la vitamina C (DT ), en la pulpa de camu camu (Myciaria dubia). Tayacaja, 2(2), 19-31. https://doi.org/10.46908/rict.v2i2.46

Torres, AY; Vidaurre, RJ. 2015. Cinética de la degradación de compuestos fenoles y antocianinas en una bebida funcional a base de arándano azul (Vaccinium corymbosum L.). Revista Ingeniería: Ciencia, Tecnología e Innovación. 2(1): 7-13.

Vargas y Vargas, ML; Figueroa, BH; Tamayo, CJA; Toledo, LVM; Moo, HVM. 2019. Aprovechamiento de cáscaras de frutas: análisis nutricional y compuestos bioactivos. CIENCIA ergo-sum, 26(2): $1-12$.

Villanueva-Tiburcio JE, Condezo-Hoyos LA, ascórbico, polifenoles totales $\mathrm{y}$ actividad antioxidante, en la cáscara de camu camu (Myrciaria dubia H.B.K. Mc Vaugh). Cienc. Tecnol. Aliment. 2010; 30(Suple 1): 151-160.

Wu, R., Frei, B., Kennedy, J. A., \& Zhao, Y. (2010). Effects of refrigerated storage and processing technologies on the bioactive compounds and antioxidant capacities of "Marion" and "Evergreen" blackberries. LWT - Food Science and Technology, 43(8): 1253-1264.

Zhou, L; Cao, Z; Bi, J; Yi, J; Chen, Q; Wu, X; Zhou, M. (2016). Degradation kinetics of total phenolic compounds, capsaicinoids and antioxidant activity in red pepper during hot air and infrared drying process. international Journal of Food Science and Technology 2016, 51, 842-853. 